$>$ II existe, dans l'hypothalamus et dans d'autres structures cérébrales comme l'hippocampe ou le striatum, des neurones spécialisés capables de détecter les variations quotidiennes des acides gras circulants. Ces neurones participent au maintien à l'équilibre de la balance énergétique en contrôlant la prise alimentaire, la sécrétion d'insuline ou la production hépatique de glucose. Les mécanismes moléculaires relayant l'effet des acides gras impliquent des récepteurs d'acides gras comme FAT (fatty acid transporter)/CD36. Toute dérégulation de cette détection centrale des acides gras peut contribuer à la mise en place des maladies métaboliques, telles que l'obésité ou le diabète de type $2 .<$

Le système nerveux central (SNC) joue un rôle majeur dans le contrôle de la balance énergétique [1, 47]. Des structures situées notamment dans notre «cerveau reptilien », comme l'hypothalamus ou le tronc cérébral, sont en effet en permanence informées du statut énergétique de l'organisme (à jeun ou nourri, autrement dit pénurie ou pléthore de calories), grâce à des signaux circulants comme des hormones (leptine, insuline, ghréline, etc.) et des nutriments (glucose, acides aminés, acides gras) (Figure 1). Ces signaux, ou plutôt la variation de leur taux sanguin, sont détectés par des neurones spécialisés [2]. Une fois les informations périphériques intégrées par le SNC, l'organisme peut s'adapter au statut énergétique en régulant les flux métaboliques (production hépatique de glucose, lipolyse, etc.) et le comportement alimentaire (début ou arrêt de la prise alimentaire), en fonction des besoins en énergie. À côté de cette régulation homéostatique (pour rappel, l'homéostasie est un ensemble de processus physiologiques permettant de maintenir certaines constantes du milieu intérieur de l'organisme nécessaires à son bon fonctionnement) de la prise alimentaire, uniquement

Vignette (Photo @ Inserm-Ariane Sharif).

\section{Rôle de la détection centrale des lipides dans le contrôle nerveux de la balance énergétique}

Valentine S. Moullé, ${ }^{1,2}$, Alexandre Picard ${ }^{1,2}$, Céline Cansell $^{1,2}$, Serge Luquet ${ }^{1,2}$, Christophe Magnan ${ }^{1,2}$

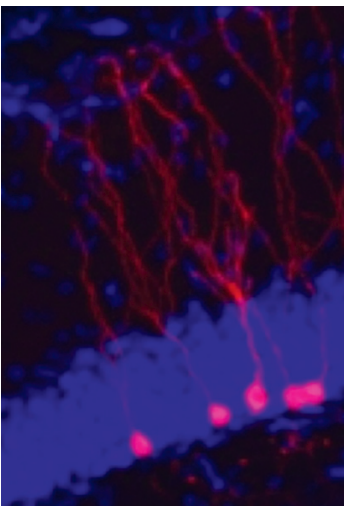

${ }^{1}$ CNRS UMR 8251, biologie fonctionnelle et adaptative, Paris, France ;

${ }^{2}$ Université Paris Diderot, 4, rue Marie-Andrée Lagroua Weill-Halle, 75205 Paris Cedex 13, France. christophe.magnan@ univ-paris-diderot. $\mathrm{fr}_{\mathrm{r}}$ valentine.moulle@gmail.com

conditionnée par un manque ou un excès de calories («j'ai faim » ou «je n’ai plus faim »), il existe aussi des régions centrales impliquées dans la réponse «non homéostatique » de la prise alimentaire (celle conditionnée par la motivation et la préférence alimentaire). Des données récentes suggèrent que, dans ces régions également, les acides gras peuvent être considérés comme des molécules informatives. Selon le concept de l'origine « centrale » des maladies métaboliques, un dysfonctionnement de ces mécanismes de détection, et notamment la détection des lipides, peut être en partie responsable d'une dérégulation de la balance énergétique et contribuer à l'apparition de l'obésité ou du diabète de type 2.

On a longtemps pensé que les acides gras, qui ne sont pas des molécules énergétiques pour les neurones, ne pouvaient pas (ou peu) franchir la barrière hémato-encéphalique et, de ce fait, ne pouvaient pas agir comme «molécule signal ». Cependant, depuis quelques dizaines d'années et, notamment, depuis les travaux d'Oomura en 1975 [3], de plus en plus de données suggèrent qu'il existe, dans l'hypothalamus, des neurones sensibles aux acides gras, et que ces derniers ont un rôle important dans la régulation de la balance énergétique, depuis la régulation de la production hépatique de glucose jusqu'au comportement alimentaire [4]. Cette revue a pour but de faire un état de l'art et de présenter les principaux mécanismes moléculaires identifiés comme des relais d'action des acides gras dans le contrôle nerveux de la balance énergétique, et leur implication éventuelle dans la dérégulation de la balance énergétique dans des situations d'apport lipidique excessif [4]. Une meilleure compréhension de ces mécanismes 


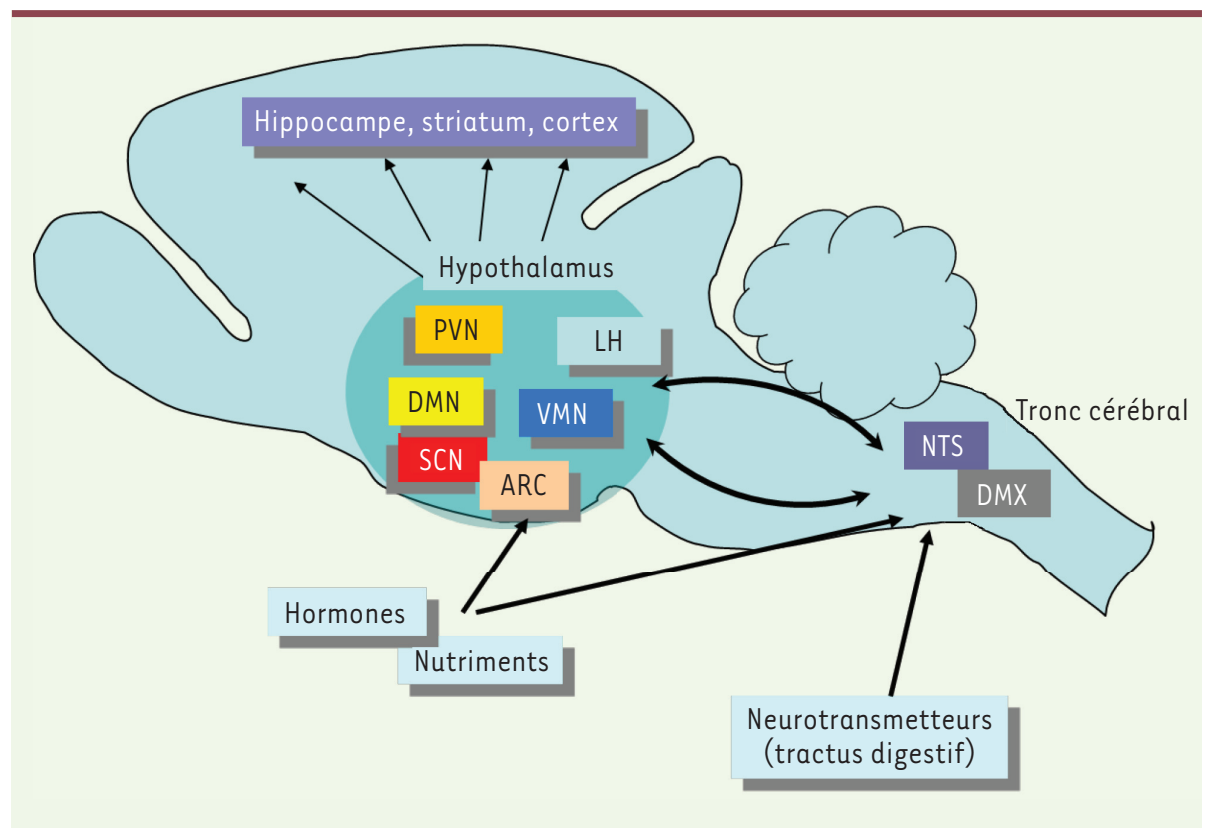

Figure 1. Modèle neuroanatomique des circuits cérébraux de régulation de la balance énergétique. Les signaux périphériques, hormones et nutriments, sont principalement détectés par le noyau arqué, tandis que les signaux du tractus digestif sont détectés au niveau du noyau du tractus solitaire. Les noyaux centraux communiquent entre eux et les informations sont finalement transmises vers le système limbique et le cortex. ARC : noyau arqué ; DMN : noyau dorsomédian ; DMX : noyau dorsal du vague; LH : noyau latéral ; NTS : noyau du tractus solitaire ; PVN : noyau paraventriculaire; SCN : noyau suprachiasmatique; VMN : noyau ventromédian.

pourrait, par ailleurs, déboucher sur de nouvelles perspectives thérapeutiques dans le traitement des maladies métaboliques.

\section{Des neurones sensibles aux acides gras dans le cerveau}

Les lipides cérébraux sont des composants essentiels des membranes et de nombreuses voies de signalisation intracellulaires. Ils représentent $50 \%$ du poids sec du cerveau, ce qui fait de ce dernier l'organe le plus riche en lipides après le tissu adipeux [5]. Des données de plus en plus nombreuses indiquent que les lipides cérébraux proviennent à la fois d'une synthèse locale et de la capture d'acides gras de la circulation sanguine, certains acides gras ayant la capacité de traverser la barrière hémato-encéphalique ( $\mathrm{BH} \varepsilon)$, notamment dans des régions où elle est plus perméable, comme l'hypothalamus. Les mécanismes par lesquels les acides gras franchissent la BHE sont encore mal connus; cela dépend de leur nature (longueur de la chaîne carbonée, degré d'insaturation, etc.). On peut cependant noter qu'ils impliquent vraisemblablement des transporteurs d'acides gras (FATP, fatty acid transporter protein). Les ARNm codant des transporteurs tels que FATP1, FATP4, ou le transporteur/récepteur FAT/CD36, ont ainsi été identifiés au niveau de neurones isolés de l'hypothalamus ventromédian, ce qui suggère la possibilité pour ces neurones de lier les acides gras à leur surface $[6,7]$. II a été aussi identifié dans ces neurones des ARNm codant des enzymes du métabolisme intracellulaire des acides gras comme l'acyl-CoA synthase (ACS), la carnitine palmitoyltransférase la et -lc (CPTla et lc) ou la uncoupling protein 2 (UCP2) [7]. L'ARNm codant l'enzyme de synthèse des acides gras (FAS) est aussi exprimé dans ces neurones [7]. II faut aussi noter que les astrocytes sont les utilisateurs principaux des acides gras, notamment le palmitate, tandis que d'autres, comme l'arachidonate, sont incorporés dans les phospholipides [8]. Ainsi, il faut intégrer dans ce réseau de communication au moins deux types cellulaires, neurones et astrocytes. Enfin, pour ajouter à la complexité, la lipopro- téine lipase ( $L P L)$ a été récemment identifiée dans les neurones (le transcrit et l'activité enzymatique) chez les rongeurs et peut aussi participer à cette signalisation [ 9 , 10]. En effet, en permettant l'hydrolyse des lipoprotéines riches en triglycérides, comme les VLDL (very low density lipoprotein) ou les chylomicrons, cette enzyme entraîne un apport local d'acides gras vers les neurones « sensibles » [11]. En dehors de l'hypothalamus, la LPL interviendrait aussi dans l'hippocampe [9] et dans le noyau accumbens, comme régulateur de la balance énergétique [12], ces structures jouant respectivement un rôle dans les processus d'apprentissage et de motivation. Ainsi, ces données mettent en lumière un effet éventuel de la détection des lipides dans des processus « non homéostatiques » de régulation de la prise alimentaire et de la balance énergétique [11].

\section{Caractérisation de neurones sensibles aux acides gras dans I'hypothalamus}

Depuis une quinzaine d'années environ, beaucoup de données ont mis en évidence que la détection hypothalamique des lipides, et plus particulièrement des acides gras, participait de façon significative à la régulation de la balance énergétique, allant de la prise alimentaire jusqu'à la sécrétion d'insuline, en passant par la lipogenèse ou la production hépatique de glucose [13]. Par exemple, une perfusion intracérébroventriculaire (Icv) d'acide oléique (un acide gras mono-insaturé) pendant $6 \mathrm{~h}$ induit, chez la souris, une diminution de la prise alimentaire et de la production hépatique de glucose [14]. Des effets similaires sont obtenus en inhibant 
l'activité de la CPTI dans I'hypothalamus, suggérant que l'oxydation des acides gras n'est pas nécessaire pour relayer les effets de ces derniers, mais que ce sont plutôt des métabolites en amont, comme les acyl-CoA, qui semblent importants [13].

Des approches plus physiologiques consistent à perfuser directement des lipides (émulsion de triglycérides ou acides gras spécifiques rendu solubles en les liant à l'albumine) dans la circulation sanguine, en introduisant à demeure un cathéter dans la carotide dont le flux est dirigé vers le cerveau, ou dans la circulation systémique (cathéter introduit dans la veine jugulaire). Ainsi, nous avons montré, chez le rat, qu'une perfusion de $48 \mathrm{~h}$ d'une émulsion de triglycérides dans la veine jugulaire induit un doublement de la concentration plasmatique d'acides gras associé à une diminution du tonus sympathique et une augmentation consécutive de la sécrétion d'insuline induite par le glucose [17]. Dans cette étude, nous avons montré que la dérégulation de la sécrétion d'insuline pouvait directement être liée à la baisse de l'activité nerveuse sympathique, car l'injection d'un agoniste pharmacologique des récepteurs adrénergiques 2 a exprimés à la surface des cellules $\beta$ pancréatiques permettait de restaurer une réponse insulino-sécrétoire normale [17]. Ce même type de réponse (baisse du tonus sympathique et réponse insulino-sécrétoire augmentée) a été également mis en évidence chez des humains perfusés avec la même émulsion de triglycérides pendant $48 \mathrm{~h}$ [18]. Enfin, une perfusion de triglycérides dans la carotide, sans modification de la concentration plasmatique de triglycérides ou d'acides gras, conduit aussi au même résultat [19]. Dans cette dernière expérience, on notait également une diminution de la prise alimentaire.

L'effet inhibiteur des acides gras sur la prise alimentaire observé expérimentalement [14] est cependant « contre-intuitif » puisque, pendant un repas, la concentration circulante des acides gras n'augmente pas, mais, au contraire, diminue [20]. À l'inverse, la prise alimentaire est fortement stimulée dans une période de reprise alimentaire après un jeûne, malgré l'augmentation du taux des acides gras libres plasmatiques. Ainsi, si l'effet «satiétogène » des acides gras est bien réel et n'est pas un biais expérimental, il nous faut admettre qu'il ne s'agit pas d'acides gras plasmatiques, mais d'acides gras d'une autre source. On peut penser aux lipoprotéines, telles que les chylomicrons, les VLDL ou même les triglycérides, dont la concentration plasmatique s'élève à la suite d'un repas [20]. Dans ce cas, il faut donc envisager une hydrolyse locale de ces lipoprotéines qui fournirait alors des acides gras aux neurones sensés les détecter. Les découvertes récentes montrant que la LPL pouvait jouer ce rôle dans le SNC suggèrent fortement qu'une telle hydrolyse locale peut effectivement se produire, et que les lipoprotéines pourraient finalement être considérées comme des molécules de signalisation ou, au moins, comme des pourvoyeuses d'acides gras aux neurones spécialisés régulant la balance énergétique $[9,10,12]$.

Ainsi, s'il est maintenant clairement établi qu'il existe des neurones sensibles aux acides gras dans le SNC (que l'on peut définir comme des neurones dont l'activité électrique est modulée [inhibée ou activée] en réponse à une variation de la concentration en acides gras), les mécanismes moléculaires relayant leurs effets font encore débat. II faut aussi avoir présent à l'esprit que les astrocytes pourraient également jouer un rôle dans cette régulation de la balance énergétique en hydrolysant les acides gras et en fournissant aux neurones des intermédiaires biochimiquement actifs, comme les corps cétoniques par exemple.

\section{Mécanismes moléculaires relayant l'effet des acides gras}

Dans les neurones sensibles aux acides gras, plusieurs études ont permis d'identifier des canaux ioniques pouvant relayer leur action. On peut citer des canaux $\mathrm{Cl}^{-}$, $\mathrm{GABA}_{\mathrm{A}}(\gamma$-aminobutyric acid-receptor $\mathrm{A})[21], \mathrm{K}^{+}, \mathrm{K}^{+}-\mathrm{Ca}^{2+}$ ou encore $\mathrm{Ca}^{2+}$ [13]. Par exemple, l'acide oléique active une population importante de neurones du noyau arqué hypothalamique, les neurones exprimant le neurotransmetteur mélanocortine (un inhibiteur de la prise alimentaire), en inhibant l'activité des canaux $\mathrm{K}^{+}$dépendants de I'ATP $\left(K_{\text {ATP }}\right)$ [24]. Par ailleurs, sur la base d'études d'électrophysiologie menées in vivo et in vitro, la proportion de neurones sensibles à l'acide oléique dans le noyau arqué a pu être quantifiée [25]. Ainsi, $13 \%$ des neurones sont excités en présence d'acide oléique, tandis que $30 \%$ environ sont inhibés chez le rat [25]. L'excitation des neurones est en partie due à la fermeture de canaux, tandis que l'inhibition implique l'ouverture de canaux $\mathrm{K}_{\text {ATP }}$ [25]. Par une technique d'imagerie calcique, des neurones sensibles aux acides gras ont pu également être identifiés dans une autre région importante de l'hypothalamus, le noyau ventromédian. Dans cette région, ce sont respectivement $43 \%$ et $29 \%$ des neurones qui sont excités ou inhibés par l'acide oléique chez le rat [7]. Dans cette population neuronale, il ne semble pas que les canaux $K_{\text {ATP }}$ jouent un rôle important pour expliquer l'effet des acides gras. II faut là encore relever aussi des différences dans les protocoles expérimentaux : en imagerie calcique, on travaille sur neurones isolés, donc en l'absence d'astrocytes, alors que les mesures électropysiologiques sont bien souvent réalisées sur des tranches de cerveau où les connexions entre les différents types cellulaires sont conservées. De plus, l'environnement en glucose semble être aussi un facteur important à prendre en considération. Ainsi, in vivo, la réponse hypothalamique des neurones « détecteurs » sera bien évidemment différente selon l'environnement hormonal et nutritionnel (à jeun ou nourri) [6].

\section{Effets des acides gras sur le contrôle nerveux de la balance énergétique via leur métabolisme intracellulaire}

La Figure 2 résume les principaux mécanismes relayant les effets des acides gras sur les neurones qui y sont 


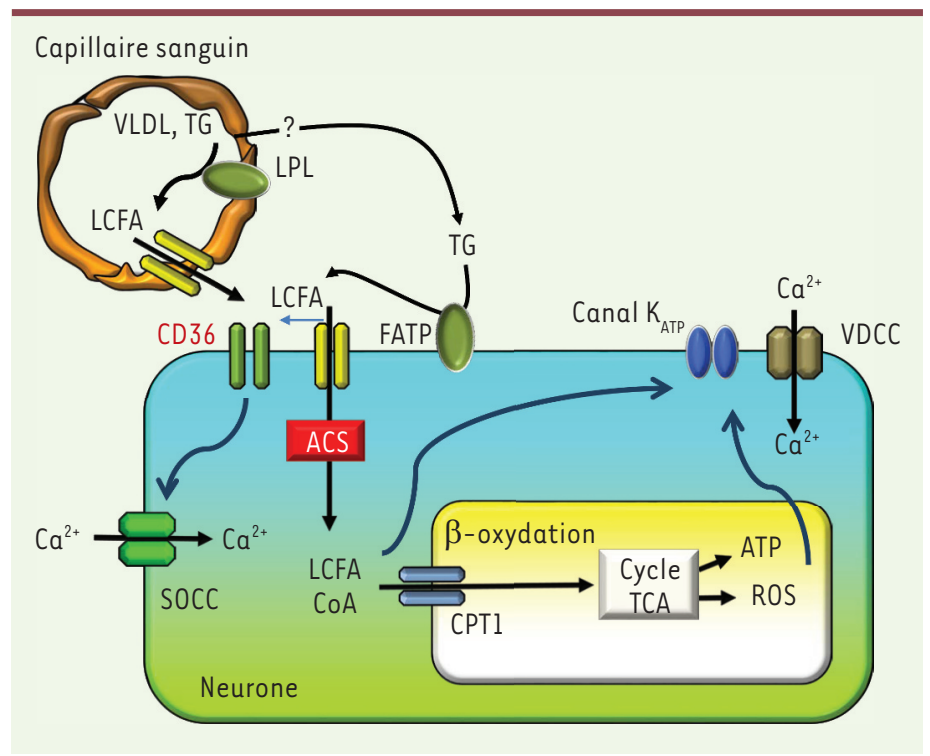

Figure 2. Modèle du neurone sensible aux acides gras. Les acides gras à chaîne longue peuvent traverser la barrière hémato-encéphalique et se lier à CD36, ou entrer dans le neurone et être métabolisés. Les triglycérides ou les VLDL peuvent être aussi localement hydrolysés dans la lumière du capillaire sanguin. ACS : acyl-CoA synthase ; CPTl : carnitine palmitoyl transférase ; FATP : fatty acid transport protein; LCFA : long chain fatty acid ; LCFA-CoA : long chain fatty acyl-COA ; ROS : reactive oxygen species; SOCC : store-operated calcium channel ; TG : triglycéride ; VDCC : voltage-dependent calcium channel; VLDL : very-low density lipoprotein.

sensibles. Comme indiqué plus haut, la machinerie enzymatique (CPTl, $A C S$, acétyl-CoA carboxylase $[A C C], F A S)$ nécessaire au métabolisme des acides gras s'exprime dans certains neurones et cellules gliales hypothalamiques [7]. Parmi les métabolites d'intérêt, le malonyl-CoA (qui est synthétisé par l'ACC) semble ainsi être un des acteurs importants capable de relayer l'effet des acides gras dans les neurones sensibles. Le malonyl-CoA est un métabolite clé qui se trouve au carrefour des voies de la glycolyse et de la $\beta$-oxydation, et il agit ainsi comme un senseur de l'état énergétique des cellules. Ainsi, quand la disponibilité en glucose augmente dans une cellule, les niveaux intracellulaires de malonyl-CoA et d'acyl-CoA augmentent en accord avec une diminution de la mobilisation des acides gras dans la voie de la $\beta$-oxydation [27]. Cette augmentation est associée à une diminution de la prise alimentaire [27]. On peut ainsi supposer qu'une augmentation du contenu intracellulaire en acyl-CoA dans les neurones peut être interprétée comme un signal de pléthore calorique et, donc, induire une baisse de la prise alimentaire. L'administration intrahypothalamique de 775 , un inhibiteur de FAS, qui induit également une augmentation de la concentration en acyl-CoA, diminue la prise alimentaire et induit une baisse du poids corporel [28]. Ceci pourrait être lié à une diminution de synthèse d'un peptide oréxigène, le NPY (neuropeptide Y). À l'inverse, l'administration par voie intracérébroventriculaire de 5-amino-imidazole-4-carboxamide ribonucléoside (AICAR), un activateur de la 5'-AMP kinase (AMPK) [48], diminue rapidement la concentration hypothalamique en malonyl-CoA et augmente la prise alimentaire [28]. Ces effets sont étroitement corrélés à l'inactivation de l'ACC consécutive à sa phosphorylation par l'AMPK. Ainsi, l'ensemble de ces données suggèrent que l'effet des acides gras sur la diminution de la prise alimentaire et la régulation de la balance énergétique nécessite leur métabolisme. Cependant, il faut avoir présent à l'esprit que ces approches pharmacologiques ont des limites. Le C75, par exemple, active également CPTl in vitro et n'agit peut-être pas uniquement via l'inhibition de la FA. L'activation de I'AMPK par I'AICAR conduit aussi à une augmentation de la synthèse des corps cétoniques par les astrocytes [32], ce qui peut aussi induire une baisse de la prise alimentaire. Ainsi, même si le métabolisme des acides gras peut être nécessaire pour relayer leur effet, on peut aussi envisager des mécanismes d'action indépendants.

On peut également signaler l'importance que pourrait jouer les espèces actives de l'oxygène dans la sensibilité centrale aux lipides, comme l'ont montré Benani et al. [33]. Il serait également intéressant de parler de ces espèces actives de l'oxygène dans les effets délétères d'un apport excessif de lipides.

\section{Effets des acides gras sur le contrôle nerveux de la balance énergétique indépendamment de leur métabolisme intracellulaire}

Les effets des acides gras sur l'activité des neurones, indépendamment de leur métabolisme, ont été notamment étudiés in vitro sur des neurones dissociés de I'hypothalamus ventromédian et exposés à de l'acide oléique $[6,7]$. Parmi les neurones « répondeurs » à l'acide gras, seuls $20 \%$ perdaient cette sensibilité lorsqu'on inhibait CPTl ou ACS [7]. Ainsi, $80 \%$ des neurones restaient sensibles, malgré le blocage de leur métabolisme intracellulaire. En revanche, l'inhibition du récepteur membranaire $F A T / C D 36$ diminuait de près de $77 \%$ la réponse des neurones « répondeurs » à l'ajout d'acide oléique au milieu de culture [6,7]. Dans cette étude, il semble que FAT/CD36 agisse principalement comme un récepteur, comme cela a été également montré au niveau des cellules de détection du goût gras localisées dans les papilles gustatives, et dans lesquelles FAT/CD36 est couplé à des canaux calcium (SOCC, store-operated calcium channel) dont l'activation induit un relargage de sérotonine [34]. Les acides gras peuvent également se comporter comme des molécules de signalisation, indépendamment de leur métabolisme intracellulaire, via des mécanismes d'acylation de protéines membranaires, qui vont ensuite induire une réponse intracellulaire. Par exemple, la palmitoylation facilite l'ancrage de protéines à la membrane 
plasmique et, en induisant un changement de leur configuration tridimensionnelle, peut activer une réaction enzymatique [35]. Des protéines membranaires, comme le TGF $\alpha$ (une protéine impliquée dans les processus d'exocytose) ou des récepteurs membranaires, comme les récepteurs adrénergiques $\alpha_{2 a}$ et $\beta_{2}$, sont des molécules palmitoylées [35]. De tels mécanismes d'acylation peuvent donc ainsi expliquer certains effets des acides gras, indépendamment de leur métabolisme intracellulaire.

D'autres protéines cytoplasmiques peuvent être aussi activées en présence d'acides gras. Il en est ainsi de la protéine kinase $C_{\Theta}\left(P K C_{\Theta}\right)$, qui s'exprime dans certaines populations neuronales des noyaux arqué et ventromédian [36]. Lorsqu'on perfuse in vivo du palmitate par voie intracérébroventriculaire chez le rat, on provoque un ancrage de la $\mathrm{PKC}_{\Theta}$ dans la membrane plasmique, ce qui a pour conséquence de l'activer et, consécutivement, de perturber la voie de signalisation hypothalamique de l'insuline [36]. On induit ainsi une résistance hypothalamique et, en conséquence, l'apparition d'une résistance à l'insuline hépatique, démontrant que l'apport excessif de lipides au niveau central perturbe le contrôle nerveux de la production hépatique de glucose, rendant l'insuline moins efficace. L'effet délétère du palmitate peut-être atténué lorsqu'on diminue l'expression de la $\mathrm{PKC}_{\Theta}$ par une approche génétique spécifiquement dans le noyau arqué [36].

Enfin, un travail tout à fait intéressant montre que les acides gras pourraient également jouer un rôle dans la plasticité neuronale hypothalamique comme décrit par Benani et al. [37].

\section{Quels neurotransmetteurs sont exprimés par les neurones sensibles aux acides gras?}

La conséquence ultime de l'activation (ou de l'inactivation) d'un neurone sera la libération d'un neurotransmetteur. Puisque la majorité des études montrent que les acides gras diminuent la prise alimentaire, on peut s'attendre à un effet sur l'activité des neurones impliqués dans ce comportement. En accord avec cette hypothèse, l'acide oléique induit effectivement l'activation (mesurée par enregistrement électrique) des neurones « anorexigènes » à mélanocortine du noyau arqué, vraisemblablement via son oxydation et l'inactivation des canaux $\mathrm{K}^{+}$dépendants de I'ATP [24]. Dans une étude déjà citée, Obici et al. [15] ont montré que l'administration intracérébroventriculaire d'acide oléique inhibe fortement la production hépatique de glucose et la prise alimentaire, et s'accompagne d'une diminution de l'expression hypothalamique du NPY, un neuropeptide « orexigène ». Dans d'autres études, la diminution de l'expression de CD36 dans l'hypothalamus ventromédian induit une baisse de l'expression de mélanocortine, se traduisant par une redistribution de la masse grasse depuis le dépôt viscéral vers la couche sous-cutanée, et l'apparition d'une résistance à l'insuline $[6,7]$. Enfin, un apport en acides gras oméga- 3 augmente la prise alimentaire dans un modèle de rat rendu anorexique à la suite de l'apparition de tumeurs [38]. Chez ces animaux, on observe une augmentation de l'expression de NPy (environ $40 \%$ ) et une diminution de l'expression de mélanocortine (environ 60 $\%)$ dans le noyau arqué, ce qui est cohérent avec l'augmentation de la prise alimentaire [38]. Cette dernière expérience souligne aussi un point important: la nature des acides gras (saturés, mono- ou poly-insaturés) peut avoir des effets différents sur le contrôle de la balance énergétique, ajoutant encore un niveau de complexité supplémentaire [39]. Ces effets peuvent s'expliquer par la production de métabolites différents en fonction de la nature des acides gras, ainsi que par une liaison à différentes protéines membranaires (CD36, GPR40 [G protein-coupled receptor 40] ou 120, par exemple). Il est à noter que la sécrétion des neurotransmetteurs (NPY ou mélanocortine) n'a jamais été étudiée dans ces différentes études.

\section{Conséquences pathologiques d'une dérégulation de la détection centrale des acides gras}

Il est maintenant bien établi, au moins dans les modèles précliniques, qu'une altération du fonctionnement de l'hypothalamus participe à l'étiologie de l'obésité [40], et que la détection centrale des lipides est altérée dans des souches de rats prédisposés à développer une obésité [7]. Ainsi, à côté des effets physiologiques des acides gras sur la régulation de la balance énergétique, on peut émettre l'hypothèse qu'un apport excessif de lipides concomitant lors de la mise en place de l'obésité peut concourir à une dérégulation de l'ensemble de la balance énergétique, via des perturbations de son contrôle nerveux [9]. Ainsi, une surcharge en lipides dans l'hypothalamus induit des modifications du tonus sympathique et une intolérance au glucose $[15,41]$, une situation caractéristique du diabète de type 2 . Un régime riche en lipides induit chez le rat une augmentation du turnover des monoamines [42] et de l'activité nerveuse sympathique. Enfin, un lien entre surcharge central en lipides et dysfonctionnement des activités neuronales peut être l'apparition d'une inflammation, comme cela a été récemment montré [43]. Parmi les métabolites des acides gras potentiellement délétères, on peut aussi mentionner les céramides [9]. Nous avons montré chez le rat qu'une augmentation de la synthèse de novo des céramides dans l'hippocampe était responsable d'une baisse de l'activité parasympathique et de l'activité locomotrice, conduisant à une obésité [9]. Chez l'homme, on a montré une augmentation de la capture des acides gras par le cerveau chez les patients atteints de syndrome métabolique [44].

\section{Conclusion}

De plus en plus de données indiquent que des neurones spécialisés sont capables de détecter les variations de concentrations circulantes de lipides dans I'hypothalamus, ainsi que dans d'autres régions comme 
I'hippocampe ou le striatum [13, 45]. Il faut bien sûr inclure dans ce système les autres molécules de signalisation d'origine hormonale (leptine, ghréline, insuline, GLP-1 [glucagon-like peptide-1]), nutritionnelle (glucose, acides aminés) ou nerveuse (afférence vagales). Ce formidable réseau de communication permet ainsi au système nerveux central d'être en permanence informé du statut nutritionnel de l'organise et de s'adapter en fonction des besoins énergétiques [47]. De nombreuses questions restent encore sans réponse : comment ces molécules circulantes gagnent-elles leurs cellules cibles et franchissent-elles la barrière hémato-encéphalique? Quel est le rôle respectif des neurones et des astrocytes? Quel est le rôle des lipoprotéines? Quels neurotransmetteurs sont libérés?

Enfin, en accord avec le concept de l'origine «centrale » du diabète de type 2 [46], une dérégulation de cette détection centrale des lipides (que l'on pourrait qualifier de « lipotoxicité centrale» par analogie à la lipotoxicité décrite dans les muscles squelettiques, le foie ou la cellule $\beta$ pancréatique) pourrait être un des facteurs déclenchant des événements menant au dysfonctionnement généralisé du contrôle nerveux de la balance énergétique [39]. Une meilleure compréhension de ces mécanismes pourrait permettre l'identification de nouvelles cibles d'intérêt thérapeutique. $\diamond$

\section{SUMMARY}

Role of brain lipid sensing in nervous regulation of energy balance Fatty acid sensitive neurons located in hypothalamus, hippocampus or striatum are able to detect daily variations of plasma fatty acid levels. Thus, these neurons play a role to regulate energy balance by controling food intake, insulin secretion or hepatic glucose production. Molecular mechanisms that mediate fatty acid effects include receptor FAT (fatty acid transporter)/CD36. Deregulation of this brain lipid sensing may be an early event leading to further dysfunction of energy balance leading to obesity and type 2 diabetes. $\diamond$

\section{REMERCIEMENTS}

Les auteurs ont bénéficié de plusieurs sources de financement: Agence nationale pour la recherche (ANR) (Lipobrain: 11-BSV1-021 01), CORDDIM Ile-de-France, European foundation for the study of diabetes (EFSD), et Société francophone du diabète (SFD).

\section{LIENS D'INTÉRÊT}

Les auteurs déclarent n'avoir aucun lien d'intérêt concernant les données publiées dans cet article.

\section{RÉFÉRENCES}

1. Luquet $S$, Magnan $C$. The central nervous system at the core of the regulation of energy homeostasis. Front Biosci (Schol Ed) 2009; $1:$ 448-65.

2. Blouet C, Schwartz GJ. Hypothalamic nutrient sensing in the control of energy homeostasis. Behav Brain Res $2010 ; 209: 1-12$.

3. Oomura $Y$, Nakamura T, Sugimori M, Yamada $Y$. Effect of free fatty acid on the rat lateral hypothalamic neurons. Physiol Behav $1975 ; 14: 483-6$.

4. Migrenne $S$, Le Foll C, Levin BE, Magnan C. Brain lipid sensing and nervous control of energy balance. Diabetes Metab $2011 ; 37: 83-8$.

5. Edmond J. Essential polyunsaturated fatty acids and the barrier to the brain: the components of a model for transport. J Mol Neurosci 2001 ; 16 : 181-93 ; discussion 215-21.
6. Le Foll C, Dunn-Meynell A, Musatov S, et al. FAT/CD36: a major regulator of neuronal fatty acid sensing and energy homeostasis in rats and mice. Diabetes 2013 ; 62 : 2709-16.

7. Le Foll C, Irani BG, Magnan C, et al. Characteristics and mechanisms of hypothalamic neuronal fatty acid sensing. Am J Physiol Regul Integr Comp Physiol 2009 ; 297 : R655-64.

8. Rapoport SI, Chang MC, Spector AA. Delivery and turnover of plasma-derived essential PUFAs in mammalian brain. J Lipid Res 2001; 42 : 678-85.

9. Picard A, Rouch C, Kassis N, et al. Hippocampal lipoprotein lipase regulates energy balance in rodents. Mol Metab $2013 ; 3: 167-76$.

10. Wang H, Astarita G, Taussig MD, et al. Deficiency of lipoprotein lipase in neurons modifies the regulation of energy balance and leads to obesity. Cell Metab 2011; $13: 105-13$.

11. Wang $\mathrm{H}$, Eckel RH. What are lipoproteins doing in the brain? Trends Endocrinol Metab $2014 ; 25: 8-14$.

12. Cansell C, Castel J, Denis RG, et al. Dietary triglycerides act on mesolimbic structures to regulate the rewarding and motivational aspects of feeding. Mol Psychiatry $2014 ; 19$ : 1095-105.

13. Picard A, Moulle VS, Le Foll C, et al. Physiological and pathophysiological implications of lipid sensing in the brain. Diabetes Obes Metab 2014; 16 (suppl 1) : 49-55.

14. Obici S, Feng Z, Morgan K, et al. Central administration of oleic acid inhibits glucose production and food intake. Diabetes $2002 ; 51: 271-5$.

15. Obici S, Feng Z, Arduini A, et al. Inhibition of hypothalamic carnitine palmitoyltransferase-1 decreases food intake and glucose production. Nat Med $2003 ; 9: 756-61$.

16. Ross RA, Rossetti L, Lam TK, Schwartz GJ. Differential effects of hypothalamic long-chain fatty acid infusions on suppression of hepatic glucose production. Am J Physiol Endocrinol Metab 2010 ; 299 : ع633-9.

17. Magnan C, Collins S, Berthault MF, et al. Lipid infusion lowers sympathetic nervous activity and leads to increased beta-cell responsiveness to glucose. J Clin Invest 1999 ; $103:$ : 413-9.

18. Magnan C, Cruciani C, Clement L, et al. Glucose-induced insulin hypersecretion in lipid-infused healthy subjects is associated with a decrease in plasma norepinephrine concentration and urinary excretion. J Clin Endocrinol Metab $2001 ; 86: 4901-7$.

19. Cruciani-Guglielmacci C, Hervalet A, Douared L, et al. Beta oxidation in the brain is required for the effects of non-esterified fatty acids on glucoseinduced insulin secretion in rats. Diabetologia $2004 ; 47: 2032-8$.

20. Ruge T, Hodson L, Cheeseman J, et al. Fasted to fed trafficking of fatty acids in human adipose tissue reveals a novel regulatory step for enhanced fat storage. J Clin Endocrinol Metab 2009 ; 94 : 1781-8.

21. Tewari KP, Malinowska DH, Sherry AM, Cuppoletti J. PKA and arachidonic acid activation of human recombinant CIC-2 chloride channels. Am J Physiol Cell Physiol $2000 ; 279$ : C40-50.

22. Honen BN, Saint DA, Laver DR. Suppression of calcium sparks in rat ventricular myocytes and direct inhibition of sheep cardiac RyR channels by EPA, DHA and oleic acid. J Membr Biol 2003 ; 196 : 95-103.

23. Oishi K, Zheng B, Kuo JF. Inhibition of Na, K-ATPase and sodium pump by protein kinase $C$ regulators sphingosine, lysophosphatidylcholine, and oleic acid. J Biol Chem $1990 ; 265: 70-5$.

24. Jo YH, Su Y, Gutierrez-Juarez R, Chua S Jr. Oleic acid directly regulates POMC neuron excitability in the hypothalamus. J Neurophysiol $2009 ; 101$ : 2305-16.

25. Wang R, Cruciani-Guglielmacci C, Migrenne S, et al. Effects of oleic acid on distinct populations of neurons in the hypothalamic arcuate nucleus are dependent on extracellular glucose levels. J Neurophysiol $2006 ; 95$ : 1491-8.

26. Migrenne S, Cruciani-Guglielmacci C, Kang L, et al. Fatty acid signaling in the hypothalamus and the neural control of insulin secretion. Diabetes 2006 ; 55 S2 : S139-44.

27. Lane MD, Wolfgang M, Cha SH, Dai Y. Regulation of food intake and energy expenditure by hypothalamic malonyl-CoA. Int J Obes (Lond) 2008 ; 32 (suppl 4) : S49-54.

28. Proulx K, Cota D, Woods SC, Seeley RJ. Fatty acid synthase inhibitors modulate energy balance via mammalian target of rapamycin complex 1 signaling in the central nervous system. Diabetes $2008 ; 57: 3231-8$.

29. Proulx K, Seeley RJ. The regulation of energy balance by the central nervous system. Psychiatr Clin North Am $2005 ; 28: 25-38$, vii.

30. Tu Y, Thupari JN, Kim EK, et al. C75 alters central and peripheral gene expression to reduce food intake and increase energy expenditure. Endocrinology 2005 ; 146 : 486-93. 


\section{RÉFÉRENCES}

31. Aja S, Landree LE, Kleman AM, et al. Pharmacological stimulation of brain carnitine palmitoyltransferase- 1 decreases food intake and body weight. Am J Physiol Regul Integr Comp Physiol $2008 ; 294$ : R352-61.

32. Blazquez C, Sanchez C, Daza A, et al. The stimulation of ketogenesis by cannabinoids in cultured astrocytes defines carnitine palmitoyltransferase I as a new ceramide-activated enzyme. J Neurochem $1999 ; 72: 1759-68$.

33. Benani A, Troy S, Carmona MC, et al. Role for mitochondrial reactive oxygen species in brain lipid sensing: redox regulation of food intake. Diabetes $2007 ; 56: 152-60$.

34. Gaillard D, Laugerette F, Darcel N, et al. The gustatory pathway is involved in CD36-mediated orosensory perception of long-chain fatty acids in the mouse. FASEB J $2008 ; 22$ : 1458-68.

35. Resh MD. Fatty acylation of proteins: new insights into membrane targeting of myristoylated and palmitoylated proteins. Biochim Biophys Acta 1999 ; 1451 : 1-16.

36. Benoit SC, Kemp CJ, Elias CF, et al. Palmitic acid mediates hypothalamic insulin resistance by altering PKC-theta subcellular localization in rodents. J Clin Invest 2009; $119: 2577-89$.

37. Benani A, Hryhorczuk C, Gouaze A, et al. Food intake adaptation to dietary fat involves PSAdependent rewiring of the arcuate melanocortin system in mice. J Neurosci $2012 ; 32: 11970-9$

38. Ramos $\varepsilon$ J, Romanova IV, Suzuki $S$, et al. Effects of omega- 3 fatty acids on orexigenic and anorexigenic modulators at the onset of anorexia. Brain Res 2005 ; 1046 : 157-64.

39. Le Stunff H, Coant N, Migrenne S, Magnan C. Targeting lipid sensing in the central nervous system: new therapy against the development of obesity and type 2 diabetes. Expert Opin Ther Targets $2013 ; 17: 545-55$
40. Velloso LA, Schwartz MW. Altered hypothalamic function in diet-induced obesity. Int J Obes (Lond) $2011 ; 35: 1455-65$.

41. Clement L, Cruciani-Guglielmacci C, Magnan C, et al. Intracerebroventricular infusion of a triglyceride emulsion leads to both altered insulin secretion and hepatic glucose production in rats. Pflugers Arch 2002; $445: 375-80$

42. Levin BE, Triscari J, Sullivan AC. Altered sympathetic activity during development of diet-induced obesity in rat. Am J Physiol 1983; 244 R347-55.

43. Cintra DE, Ropelle $\varepsilon R$, Moraes JC, et al. Unsaturated fatty acids revert dietinduced hypothalamic inflammation in obesity. PLoS One 2012; 7 : e30571.

44. Karmi A, lozzo P, Viljanen A, et al. Increased brain fatty acid uptake in metabolic syndrome. Diabetes 2010; $59: 2171-7$.

45. Contreras C, Lopez M. Ceramide sensing in the hippocampus: the lipostatic theory and Ockham's razor. Mol Metab 2014; $3: 90-1$.

46. Elmquist JK, Marcus JN. Rethinking the central causes of diabetes. Nat Med $2003 ; 9: 645-7$.

47. De Vadder F, Mithieux G. Contrôle de la glycémie par l'axe nerveux intestincerveau. Med Sci (Paris) $2015: 31: 168-73$.

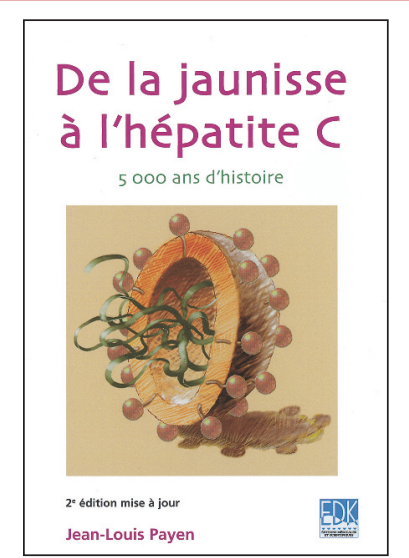

ISBN : 978-2-8425-4136-1 128 pages a jaunisse est un symptôme facilement identifiable ; il paraissait bien naturel que l'homme, confronté à une modification de la couleur de ses yeux et de sa peau ait de tous temps recherché les causes de cette transformation.

II n’est donc pas surprenant que le premier traité de médecine, écrit 3000 ans avant J.C. par un médecin sumérien, décrive déjà la jaunisse. À chaque époque de l'histoire de la médecine, les praticiens, influencés par les concepts médicaux de leur temps, attribuèrent une ou plusieurs explications particulières à ce symptôme. Ainsi, du démon Ahhâzu des Sumériens à la sophistication des biotechnologies qui permirent la découverte du virus de l'hépatite $C$, le lecteur cheminera sur une période de 5000 ans au travers des différents continents. Ici encore, l'histoire se révèle une formidable source de réflexion : le foie souvent impliqué dans l'apparition des jaunisses est-il le siège de l'âme?

Les expérimentations humaines chez des volontaires ou chez des enfants handicapés mentaux étaient-elles justifiées pour permettre la découverte des virus des hépatites?

Le formidable développement de la transfusion sanguine, des vaccinations, mais aussi de la toxicomanie explique-t-il les épidémies d'hépatites du XXe siècle?

Autant de questions qui sont abordées dans ce livre passionnant et accessible à tous.

\section{À retourner à EDK, 109, avenue Aristide Briand - 92541 Montrouge Cedex}

UU Tél. : 0141177405 - Fax : 0143293262 - E-mail : edk@edk.fr

NOM :

Prénom

Adresse :

Code postal :

Ville :

Pays :

Fonction :

Je souhaite recevoir l'ouvrage De la jaunisse à l'hépatite $\mathbf{C , ~} \mathbf{5} \mathbf{0 0 0}$ ans d'histoire : $12 €+3 €$ de port $=\mathbf{1 5} € \mathbf{T T C}$

en ................ exemplaire, soit un total de ................................... €

U Par chèque, à l'ordre de $\mathbf{E} \mathbf{D} \mathbf{K}$

$\square$ Par carte bancaire : $\square$ Visa $\square$ Eurocard/Mastercard

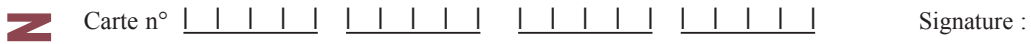

Date d'expiration: $\quad|\quad| \quad|\quad|$

$\mathrm{N}^{\circ}$ de contrôle au dos de la carte :

\section{$1|1|$}

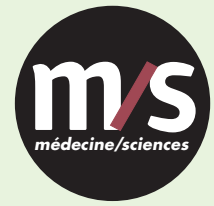

Tarifs d'abonnement $\mathrm{m} / \mathrm{s}-2015$

Abonnez-vous

à médecine/sciences
$>$ Grâce à $m / s$, vivez en direct les progrès des sciences biologiques et médicales

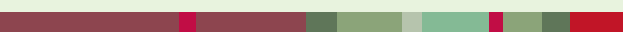

Bulletin d'abonnement page 453 dans ce numéro de $\mathrm{m} / \mathrm{s}$

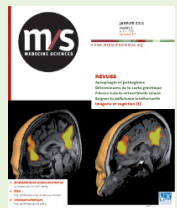

